DECISION MAKING QUALITY OF YOUNGER

AND OLDER ADULTS IN FAMILIAR

AND UNFAMILIAR DOMAINS

by

ERNEST WAYDE

\begin{abstract}
A THESIS
Submitted in partial fulfillment of the requirements

for the degree of Master of Arts

in the Department of Psychology

in the Graduate School of

The University of Alabama
\end{abstract}

TUSCALOOSA, ALABAMA 
Copyright Ernest Wayde 2010

ALL RIGHTS RESERVED 


\begin{abstract}
The older adult population of the United States is expected to increase substantially in the next twenty years. Working memory, one of the cognitive resources crucial for decision making, declines with age. The decline in working memory might have a negative effect on the quality of purchasing decisions that older adults make.

However, older adults have greater experience than younger adults and may be able to use past knowledge or schemas to assist them in decision making. By using a schema in familiar situations, older adults can conserve cognitive resources and thereby make high quality decisions.

In this study, age-related changes in decision-making were examined as a function of age and familiarity. Participants consisted of 55 younger adults and 46 older adults. Participants were presented with purchasing scenarios and asked to choose between two products described in the scenario. In each instance, inferencing was required to select the optimal option from the two choices. Domain knowledge about the to-be-purchased product was also manipulated.

Results support the hypothesis that younger adults generally make good quality decisions regardless of the domain while older adults make better quality decisions in the familiar domain as opposed to the unfamiliar domain.

Current findings suggest that although adult decision making ability is compromised with age, older adults are able to use existing domain knowledge of a specific area to compensate for the lack of cognitive resources in order to make high quality decisions.
\end{abstract}




\section{LIST OF ABBREVIATIONS AND SYMBOLS}

\begin{tabular}{ll}
$\alpha$ & Cronbach's index of internal consistency \\
$F$ & Fisher's $F$ ratio: A ratio of two variances \\
$M$ & Mean: the sum of a set of measurements divided by the number of measurements \\
& in the set \\
$p$ & Probability associated with the occurrence under the null hypothesis of a value as \\
$r$ & extreme as or more extreme than the observed value \\
$t$ & Pearson product-moment correlation \\
$\eta^{2} \mathrm{p}$ & Computed value of $t$ test \\
$\mathrm{n}$ & Partial eta squared \\
$S D$ & Sample size for group \\
ANOVA & Analysis of Variance \\
$<$ & Less than \\
$=$ & Equal to \\
MMSE & Mini Mental Status Exam \\
HOA & Home Owners Association \\
WAIS-R & Wechsler Adult Intelligence Scale-Revised \\
MRQ & Multiple Resources Questionnaire \\
kb & Mean Squared Error \\
\hline
\end{tabular}




\section{ACKNOWLEDGMENTS}

I would like to thank my advisor and thesis chairperson, Sheila Black, for her advice, guidance and knowledge throughout the entire process. I would also like to thank the members of my committee, Debra McCallum and Mark Klinger, for their input concerning the design, implementation and write up of this project. Finally, I would like to thank all the participants who participated in this research. Without them, this would not have been possible. 


\section{CONTENTS}

ABSTRACT ................................................................................. ii

LIST OF ABBREVIATIONS AND SYMBOLS ................................. iii

ACKNOWLEDGMENTS ............................................................ iv

LIST OF TABLES ..................................................................... vi

LIST OF FIGURES ................................................................ vii

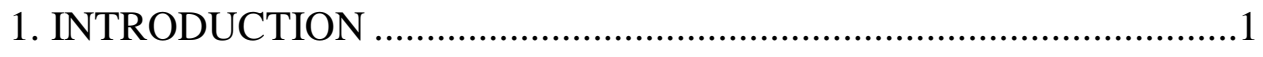

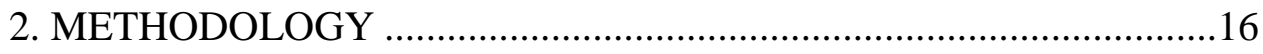

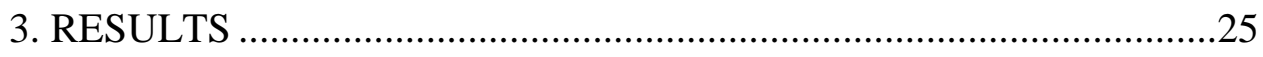

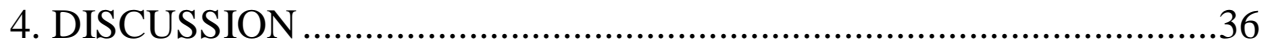

5. CONCLUSION ..................................................................... 42

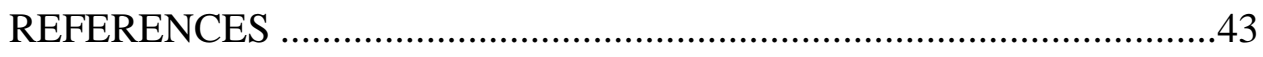

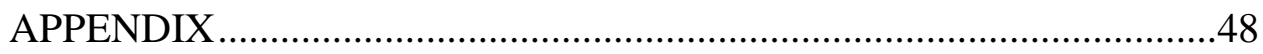




\section{LIST OF TABLES}

1. Participant Mean and Standard deviation (SD) for house and cell phone purchases and

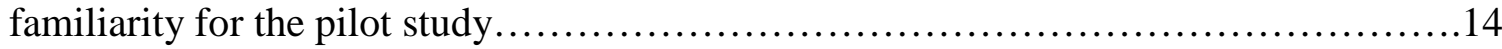

2. Participant Mean and Standard deviation (SD) for house and cell phone purchases and

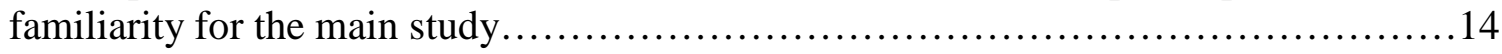

3. Participant Mean and Standard deviation (SD) for age, vocabulary scores and Trail

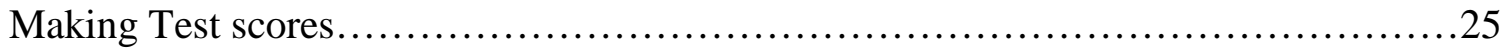

4. Participant Mean and Standard deviation (SD) for decision making quality in implicit familiar and unfamiliar scenarios

5. Participant Mean and Standard deviation (SD) for decision making quality of familiar and unfamiliar purchasing scenarios in the explicit condition...

6. Beta Coefficients, Beta significance, $R^{2}$ change, $F$ change and Significance $F$ change of percentage correct in unfamiliar decision making scenarios for age after controlling for

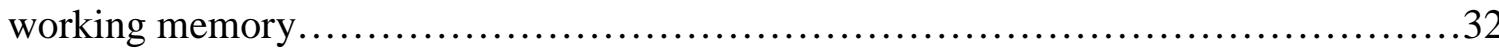

7. Participant Mean and Standard deviation (SD) for decision making quality in unfamiliar implicit scenarios with a median split of Need for Cognition scores.

8. Coefficients, significance, Increment in $\mathrm{R}^{2}, \mathrm{~F}$ change and Significance of $\mathrm{F}$ change for percentage correct in unfamiliar decision making scenarios affected by age after controlling for working memory and Need for cognition.

9. Intercorrelations Amongst Unfamiliar decision scores, Need for Cognition, Trail B and

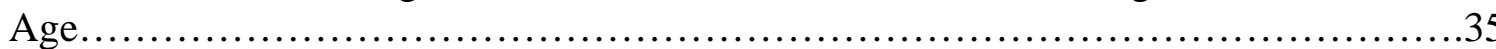




\section{LIST OF FIGURES}

1. Younger and Older adult average scores for the individual scenario ratings.................31 


\section{INTRODUCTION}

The older adult population of the United States is expected to increase substantially in the next twenty years. Older adults, a group categorized by adults aged 65 and older, will number 70 million by 2030 (Polyak, 2000). Due to decreases in birth rates and increases in life expectancy (Rowe \& Kahn, 1998), older adults will become the largest consumer group in the United States. Older adults will have a lot of buying power (Polyak, 2000) and will be making important decisions about healthcare, housing, and finance.

Unfortunately, as individuals' age, cognitive functioning is decreased, thus requiring older adults to use a greater proportion of their limited cognitive resources in the decision making process (Moye \& Marson, 2007). As a result of limited cognitive resources, a number of studies have shown that older adults become cognitive misers (Morris, Woo, and Singh, 2005) relative to younger adults. Cognitive resources are reserved for decisions that not only require careful deliberation but will also have a big impact on the lives of the older adult. One of the goals of this study was to determine the extent to which older adults rely on heuristics rather than thoughtful analyses in making purchasing decisions, due to age-related changes in cognitive resources. It was predicted that older adults would be more likely than younger adults to rely on heuristics and peripheral cues as opposed to thoughtful deliberation in making purchasing decisions. This decision making technique might make older adults more vulnerable to fraudulent predators, financial scams, and questionable marketing tactics (Yoon et al., 2005). Hess, Germain, Rosenber, Leclerc and Hodges, (2005) argue that older adults might allocate less resources to decision making tasks because of declines in working memory. Due to the decline in 
working memory, elaborative processing is more taxing for older adults than for younger adults. Because working memory is so important for decision making and there are documented agerelated changes in working memory, the next section focuses on age-related changes in working memory.

\section{Working Memory}

Older adults have a smaller working memory span than younger adults and are therefore less able to consider and analyze information required to make a decision than younger adults. Recent research has shown that as the amount of information processing (deliberation and indepth analysis) and the complexity of the decision required increases, older adult comprehension (the ability to understand information) of the information decreases because their reasoning and memory skills are exhausted (Finucane, Mertz, Slovic, Scholze and Schmidt, 2005).

Baddeley and Hitch (1974) developed a model that focuses on the structure of working memory within the information processing system. Their model has had a tremendous impact on the conceptualization of working memory. According to their model, one component of working memory that is important for decision making is the component often referred to as the central executive. The central executive allows human organisms to engage in cognitive activities called executive processes, which include exercising cognitive control, reasoning ability, and integration. The central executive is the part of working memory most vulnerable to the effects of aging (Van der Linden \& Bredart, 1994). Age related declines in working memory, and the effect these changes have on cognitive processes, have been discussed extensively in the literature (Brébion, Ehrlich and Tardieu, 1995; Estevez and Calvo, 2000). The next section focuses on the impact of age-related changes in working memory resources on the decision making ability of older adults. Specifically, the next section utilizes a decision making model to 
predict the conditions under which older adults will use a heuristic versus elaborative processes to make decisions.

\section{Elaboration Likelihood Model}

The Elaboration Likelihood Model is a decision making model that is useful in explaining decision making in older adults. The Elaboration Likelihood Model proposes that when people make decisions, they will use one of two routes: the central or the peripheral route (Petty \& Cacioppo, 1984). The route chosen for decision making depends on the amount of resources available. The central route requires more resources than the peripheral route. To explain further, in the central route of elaboration, persuasion occurs more often when the participant is motivated and has the mental capability to analyze and process the information provided (Te'Teni-Harari, Lampert, and Lehman-Wilzig, 2007). Individuals using the central route will carefully and thoughtfully analyze the information and the detail related to the decision (Christensen, Ascione, and Bagozzi, 1997). On the other hand, when people use the peripheral route, they are relying on short cuts or heuristics to make a decision rather than careful elaboration (Cacioppo \& Petty, 1984).

In the Elaboration Likelihood Model, the peripheral route requires significantly less elaboration of the message details than the central route and is more often used when the message has little relevance or motivation for the receivers. A person then makes the decision based on peripheral cues or heuristics such as affect cues (e.g., source likability), source attractiveness, and message length. Because the peripheral route requires fewer cognitive resources it does not put as much strain on the limited cognitive resources of older adults as the central route of elaboration. When people use the peripheral route, they often make use of existing schemas from past experiences coupled with superficial knowledge to make a decision 
(Cacioppo \& Petty, 1984). To reiterate, it might be the case, that older adults relative to younger adults, will be more likely to use the peripheral than the elaborative route when making decisions because of age-related changes in working memory resources. However, as the peripheral route of elaboration does not require older adults to engage in thoughtful consideration and analysis of the data, important information pertinent to the decision making process may be overlooked.

\section{Information and Detail}

Careful analysis and attention to detail are important with regard to a number of domains, in particular, with regard to healthcare. For example, as older adults reach and pass the age of retirement, they will be required to select their own healthcare and Medicare plans, and to make decisions about housing arrangements and manage their own finances. Traditionally, about eighty-two percent of older adults seek advice from a family member when making such decisions (Sorce et al., 1989), but the final decision is still left up to the older adult. As analysis of complex data is required and a choice must be made between numerous options, older adults should use the central route to carefully analyze all of their options before making a decision. However, recent research has shown that in decision making, older adults prefer less choice and seek less information when making decisions (Reed, Mikels and Simon, 2008). In the study by Reed et al., researchers asked younger and older adults to indicate the number of options they would like to be given when making choices about items such as cars, apartments, hospitals, and physicians. Results show that older adults prefer significantly less choice and detail than younger adults across all domains and this preference strengthened with age.

In fact, other researchers, such as Finucane et al. (2002), have shown that older adults themselves believe they are less able to analyze and make decisions when dealing with complex information such as charts and tables. When comparing the amount of information that college 
age students and older adults use in making purchasing decisions about cars and computers, Johnson (1990) found that college age students used more information and spent less time on each piece of information than older adults.

\section{Age, Inhibition and Decision Making}

Even though it seems older adults prefer to use less information when making decisions, there is a question as to whether they are able to inhibit irrelevant information when presented with relevant and irrelevant information associated with making a decision (Hasher \& Zacks, 1979). Older adults seem to be affected by extraneous material to a far greater degree than younger adults (Connelly, Hasher and Zacks, 1991). This inability to filter irrelevant information could have a negative effect on decision making for older adults when irrelevant information is provided in advertising. For example, advertisements frequently include lures which provide little information about the quality of the product but are included to persuade the customer to purchase the product. If older adults are unable to suppress the influence of such irrelevant information, they will be more vulnerable to fraudulent or predatory advertising techniques that take advantage of their inability to be selective in information processing. An additional problem for older adults is that they have a problem suppressing no-longer-relevant thoughts and information (Hartman \& Hasher, 1991) from past decisions and when facing a new decision that requires new information and new ideas.

Experience and Decision Making

One approach to improve older adults' decision making ability is for older adults to use a strategy approach to determine the circumstances under which it would be appropriate for them to use cognitive resources and when to rely on past knowledge (Mata, Schooler and Rieskamp, 2007). Unfortunately as individuals' age, it seems cognitive decline also leads to deficits in 
adaptive strategy selection (Lemaire, Arnaud and Lecacheur, 2004), resulting in older adults' being further disadvantaged by not being able to select the appropriate strategy to make a decision.

One reason that it might be difficult for older adults to select an appropriate strategy is that they often fail to comprehend all of the details associated with complex decisions. They especially have difficulty comprehending written material in which the features associated with a product choice are not explicitly stated. Before older adults can select the appropriate strategy for decision making they have to be able to comprehend product related information. Thus, it is important to examine older adults' ability to understand information that is only implicitly rather than explicitly stated.

\section{Inferences}

As indicated earlier, older adults struggle with the lack of cognitive resources required for thoughtful decision making. Therefore, older adults are particularly disadvantaged relative to younger adults when information is not explicitly stated and inferences have to be made (Hamm \& Hasher 1992). Till and Walsh (1980) have concluded that older adults are less likely to generate inferences spontaneously when transferring information from short-term to long-term memory. On the basis that older adults lack inferencing ability during encoding, Cohen (1981) showed that older adults perform poorly on decision making tasks that require inferencing.

With a decreased ability to draw inferences from implicitly stated information in the decision making process, older adults may miss important information that is not explicitly stated but implicitly inferred. The inability of older adults to draw inferences may result from age differences in working memory (Zacks \& Hasher, 1988). As indicated earlier, working memory capacity decreases with the advancement of age in older adults. 
Brébion, Ehrlich and Tardieu (1995) theorize that older adults' problems with tasks that require self-initiated processing (e.g., inferencing) stem from age-related declines in working memory resources. Wright and Newhoff (2002) have done research that confirms Brebion et al.'s theory. They found in their research that older adults demonstrate a significantly stronger relationship between working memory and comprehension than young adults. It would seem, therefore, that age-related problems in inferencing are due primarily to the deficit in working memory resources that older adults suffer as a result of aging.

Further evidence of the relationship between working memory and inferencing is provided by Estevez and Calvo (2000) who performed a study to examine whether the time for inferencing was affected by individual differences in memory span. They theorized that inferencing is an elaboration task that requires time, effort, and resources and this task will accordingly be constrained by working memory resources. The results of their study show that the construction of implicit information is positively correlated within working memory span.

Inferencing ability is also affected by domain knowledge. Initially, there was some debate about whether the cognitive processes associated with inferencing were domain-free or domain specific (Roberts, Welfare, Livermore and Theadom, 2000), but further research has shown the effect of domain knowledge on the cognitive inferencing process. Past experiences may allow for the faster recognition of key themes and underlying rules that relate to a specific domain (Roberts et al.). It is therefore easier and faster to comprehend information associated with a domain with which you have existing knowledge.

\section{Domain relevance}

As indicated earlier, older adults have the advantage of domain knowledge that can offset cognitive resource limitations when making decisions about familiar domains. Staudinger and 
Baltes (1996) have suggested that older adults carry greater bodies of knowledge than younger adults and are able to use this advantage in the decision making process. Results of their study show domain experience facilitates cognitive functioning to a higher degree in older adults than younger adults in certain conditions. More recently, research by Leclerc and Hess (2007) has shown that social experience and domain knowledge allows for the creation of an expertise framework with which older adults are able to better solve problems and make decisions. An example of such a decision occurs when older adults have to make decisions about their health care.

Meyer, Talbot, and Randali (2007) found that older adults preferred to make more immediate decisions about cancer treatments than younger adults and that familiarity with treatment knowledge was the best predictor of immediacy of treatment knowledge. Similarly, a study conducted by Johnson and Drungle (2000) demonstrated the way in which familiarity facilitates decision making ability among older adults. In this study, participants were asked to select various types of medication. Participants had to search through four medication labels before making the choice. It was expected that older adults had more experience with pain medication than younger adults. When making decisions about over the counter medication older adults took less time deciding what pain medication to purchase as opposed to the time spent making a decision about other kinds of medication with which they had little experience.

Older adults' lack of experience with selecting other medications meant they had to carefully and thoughtfully analyze the information on the labels, taxing their limited cognitive resources and slowing down their decision making process. As mentioned earlier, the decrease in response time for older adults' selection of pain medication was attributed to the fact that older adults have enough past experience with pain relievers and as such did not have to spend much 
time or cognitive resources carefully analyzing the medication labels as they could rely on this past knowledge to help in the decision making process. While this shortcut in decision making may seem to be helping older adults, it could also be dangerous to older adults in their decision making capacity.

When older adults rely too heavily on familiarity, they may not consider each situation uniquely. For example, an older adult who has had to take medication on multiple occasions for pain relief may be tempted to rely on his or her pain medication schema when experiencing new pain symptoms that require new medication. However, relying too heavily on the pain medication schema could present a problem. The current pain problem may not be the same as the initial situation from which the experience was gained. However, the same product choices might be made even though this new situation is different and requires new choices.

\section{Current Study}

In the present study, I examined the quality of decisions made by older adults when compared to younger adults in familiar and unfamiliar domains. I also examined the way in which age related changes in inferencing ability affect older adults' ability to make good purchasing decisions. Participants were given four purchasing scenarios, each scenario containing two options. Participants where then asked to choose the option which provided the best value for money. Each participant received two purchasing scenarios in which some inferencing was required and of those two inferencing conditions, one purchasing condition was familiar and the other was unfamiliar to the participant.

As indicated earlier, there are two routes to persuasion, the peripheral and central route. There are several reasons to think that older adults will be more likely to choose the peripheral route than younger adults. First, there is evidence of an age related decline in working memory. 
Second, older adults are cognitive misers who prefer to use as little cognitive resources as possible when making decisions. Because they are cognitive misers, older adults prefer fewer choices and less detail than younger adults when making decisions. Third, there is evidence that older adults prefer to make decisions in domains with which they are familiar because they can use their knowledge from similar past experiences to make the decision making process easier and less cognitively reliant. Thus, older adults might be more tempted than younger adults to rely on a schema when making a decision, rather than careful analysis.

I predicted that participants making decisions based on the elaborative route would choose the high quality product over the low quality product when the cost of the two products was the same. On the other hand, if participants were using the peripheral route, they would be more likely to make decisions based on "catch phrases" in the advertisement, designed to lure the participant, rather than making decisions based on the quality of the product.

\section{Hypotheses}

This study attempted to explore the interaction between age and familiarity as these variables are usually considered together in real life decision making situations.

Hypothesis 1: It was predicted that younger adults would rate a high quality product more favorably than a low quality product with greater consistency than older adults.

In a familiar domain, older adults should have the ability to use an existing schema or previous knowledge when making a decision. However, no such knowledge or schema would exist in an unfamiliar domain, requiring older adults to use self initiated processes and careful analysis of the information available to make the decision. Older adults do not perform as well as younger adults on tasks that require self initiated processes (Brébion, Ehrlich and Tardieu, 1995: Wright $\&$ Newhoff, 2002). 
Hypothesis 2: It was predicted that in familiar domains younger and older adults would consistently rate a high quality product more favorably than a low quality product. However, when older adults were presented with an unfamiliar domain, they would be less likely to rate high quality products more favorably than low quality products. Younger adults, on the other hand, would consistently rate a high quality product more favorably than a low quality product regardless of domain.

A house purchasing scenario was used to represent a familiar domain for older adults and an unfamiliar domain for younger adults, while a cell phone purchasing scenario was used to represent a familiar domain for younger adults and an unfamiliar domain for older adults. In a familiar domain, older adults should still be able to make good quality decisions by making use of existing decision making schemas coupled with the superficial knowledge. This combination should allow older adults to consider more information in the decision making process by relying on previous knowledge and analysis of a similar decision. In an unfamiliar domain, older adults would not have the benefit of this previous knowledge or schema and would either use limited cognitive resources or heuristics and simple affect cues. It has been shown that older adults prefer to use heuristics and simple affect cues if the decision does not have a direct impact on them or if the information strains working memory resources.

Hypothesis 3: It was predicted that the relationship between age and decision making performance would be mediated by working memory. As stated earlier, the central executive which allows individuals to engage in cognitive activities is a part of working memory that is most vulnerable to the effects of aging (Van der Linden \& Bredart, 1994). In addition, older adults have smaller working memory spans than younger adults and this affects their decision 
making performance. Therefore, the relationship between age and decision making performance should be mediated by working memory.

Hypothesis 4: It was predicted that Need for Cognition would modulate age effects in this study. That is, age differences should be smaller on the decision making task when older adults have a high need for cognition relative to when older adults have a low need for cognition. This is because high need for cognition refers to receiving a high degree of enjoyment from analytical thinking. Although older adults in general are cognitive misers it is expected that older adults with high need for cognition will enjoy carefully analyzing and comparing the features associated with products and will thus approach the task like younger adults. Thus, even though older adults in general will be more likely to use the peripheral rather than the elaborative route, older adults with high need for cognition should use the elaborative route fairly consistently, given that they enjoy elaborative processing.

\section{Pilot Study}

A pilot study was performed first to confirm that the house purchasing scenario was familiar to older adults and the cell phone purchasing scenario was familiar to the younger adults. Twenty older adults and 20 younger adults were asked to answer questions in a survey. They were asked:

1. If they had ever bought a cell phone before and how many times they have performed this task. Participants answered with a "yes" or "no" and indicated an estimation of how many times they had performed the task when applicable.

2. In addition, participants indicated how much information they knew about purchasing a cell phone. This question was answered on a seven point scale in which "1" represented no information and a score of "7" represents an expert. 
Participants were asked the same questions about purchasing a house. Twenty Older adults were recruited from the surrounding community and through appeals to retirement homes. Older adults were asked the same questions as the younger adults in the form of a paper survey. Compensation of $\$ 5$ was given to older adults. Younger adults were compensated with course credit. Paired $t$-tests were conducted to determine if the study assumptions were correct. The results of this information are displayed in the results section. The results of the paired $t$-tests show significant results that verified the premise of the hypothesis in that younger adults were familiar with the task of purchasing cell phones and unfamiliar with the task of purchasing a house while older adults were familiar with the task of purchasing a house and unfamiliar with the task of purchasing a cell phone.

\section{Pilot Study Results}

As indicated earlier, a pilot study was conducted to examine the assumptions that the house purchasing scenario would be more familiar to older adults than the cell phone purchasing scenario and alternatively, the cell phone purchasing scenario would be more familiar to younger adults than the house purchasing scenario. A paired $t$-test analysis was conducted on the data from the 20 older adults (mean age $=76.50, S D=8.33$ ) and 20 younger adults (mean age $=$ $17.90, S D=1.25$ ) who participated in the study. The means and standard deviations are displayed in Table 1.

As one can see from Table 1, younger adults, purchased cell phones significantly more times than houses, $t(19)=7.96, p<.01$. Younger adults also indicated more familiarity with the cell phone purchasing scenario than with the house purchasing, $t(19)=7.19, p<.01$. For older adults, the reverse was true. As one can see from Table 1, older adults purchased houses significantly more times than cell phones, $t(19)=2.94, p<.01$. Older adults also indicated 
more familiarity with the house purchasing scenario than with the cell phone purchasing scenario, $t(19)=2.90, p<.01$. These results support the main study premise that older adults are familiar with houses and purchasing houses but unfamiliar with cell phones and purchasing cell phones.

Table 1

Participant Mean and Standard deviation (SD) for house and cell phone purchases and familiarity for the pilot study

\begin{tabular}{lccccc}
\hline & \multicolumn{2}{c}{ Younger adults $(\mathrm{n}=20)$} & & \multicolumn{2}{c}{ Older Adults (n=20) } \\
\cline { 2 - 3 } \cline { 5 - 6 } & Mean & SD & & Mean & SD \\
\hline House purchasing & $<.01$ & $<.01$ & & 1.60 & 1.14 \\
Cell phone purchasing & 3.00 & 1.69 & & 1.10 & 1.33 \\
House purchasing familiarity & 1.65 & 1.23 & & 3.90 & 2.18 \\
Cell phone purchasing familiarity & 4.85 & 1.66 & & 2.70 & 2.25 \\
\hline
\end{tabular}

Table 2

Participant Mean and Standard deviation (SD) for house and cell phone purchases and familiarity for the main study

\begin{tabular}{lccccc}
\hline & \multicolumn{2}{c}{ Younger adults(n=55) } & & \multicolumn{2}{c}{ Older Adults(n=46) } \\
\cline { 2 - 3 } \cline { 5 - 6 } & Mean & SD & & Mean & SD \\
\hline House purchasing & .02 & .14 & & 2.0 & 1.94 \\
Cell phone purchasing & 3.65 & 1.97 & & 1.24 & 1.54 \\
House purchasing familiarity & 1.95 & 1.42 & & 4.0 & 2.10 \\
Cell phone purchasing familiarity & 5.33 & 1.35 & & 2.85 & 1.98 \\
\hline
\end{tabular}


In addition to collecting information about the familiarity of the various products in a pilot study, I also collected this same data in the main study. The results are consistent with the results obtained from the pilot study. This data is summarized in Table 2. 


\section{METHODOLOGY}

\section{Participants}

A power analysis was conducted based on the extant literature to determine the number of participants needed so that the power to detect significant findings would be $80 \%$ and the chance of committing a Type I error would be at 5\%. Based on the previous literature, I assumed that the effect size in the current study would be small; the power analysis indicated that 84 participants (42 per group) would be needed. My sample size exceeded the recommended number of participants.

A total of 101 participants consisting of younger and older adults participated in the study. Fifty five younger adults, (Mean age $=18.71$ years, $S D=1.45$ ) were obtained using the student research pool at the University of Alabama. Younger adult participants received research credit for their Psychology 101 course. Older adults (Mean age $=73.63$ years, $S D=8.09$ ) were defined as adults at or over the age of 65 . The 46 older adult participants were recruited from the surrounding community through appeals to retirement homes, assisted living facilities and senior citizen community centers. Compensation of $\$ 10$ was provided for older adult participants. All older participants in this study met the minimum required score of 24 on the Mini Mental Status Exam (MMSE), a dementia screening test. A score of 24 or above indicates that the person does not suffer from dementia. The study was self paced and took approximately 25 minutes for younger adults to complete and approximately 35 minutes for older adults to complete. 


\section{Design}

The experimental design was a 2 Age (Young vs. Old) x 2 Familiarity (Familiar vs. Unfamiliar domain) mixed factorial design. Age was a between-subjects variable. Familiarity was manipulated within-subjects. The dependent variable was percent correct. As will be explained in more detail in the Materials section, on each trial, participants received two products which cost the same but varied in quality. A decision was considered correct if a participant chose the higher quality option over the lower quality one.

\section{Materials}

Four different trials were created. During a given experimental session, participants received each of these four individual purchasing scenario trials. Within each purchasing trial, participants had 2 options from which to choose. The information was displayed on the computer screen in 20-point Times New Roman font against a white background. Examples of the scenarios are included as Appendix A.

On each trial, participants received purchasing options which varied in quality but ultimately cost the same amount of money. The options were presented in a two-column table format, and each column in the table represented a separate option. As one can see in Appendix A, the product label was always written in 20 PT. bold font and was positioned at the top of each column. Features (in regular 20 point font) associated with each product were listed below the product labels. As indicated before, a participant's response would be considered correct if he or she chose the higher quality option over the other lower quality option.

Both familiarity and explicitness of information were manipulated in this study. First turning to the familiarity manipulation, for each participant, half of the scenarios focused on familiar products and the other half of the scenarios focused on unfamiliar products. Likewise, 
with regard to the explicitness of the information, for each participant, half of the scenarios provided explicit information about the features of the product and required little inferencing (the first two scenarios in Appendix A); the other half of the scenarios could only be fully comprehended if the participant "read between the lines" and made more complex inferences about the information presented in the scenario (the last scenario in Appendix A).

\section{Manipulation of Explicitness of Information}

To explain the explicitness manipulation in more detail, in the explicit condition, participants had to make choices about items that clearly differed in quality. The third scenario presented in Appendix A provides an example of a scenario in the explicit cell phone condition. As one can see, one option is superior to the alternative on numerous dimensions. Moreover, the price is the same for the two products. Thus, one can easily choose the better of the two options in this instance. Because choice selection was so easy and straightforward in the explicit condition, I expected ceiling effects. Thus, the explicit condition was used as a manipulation check and wasn't included in the primary analyses. I expected both younger and older adults to choose the higher quality option reliably in the explicit condition.

Examples of the implicit condition are displayed in the first two scenarios in Appendix A. As one can see from the first scenario in Appendix A, on the surface one might assume that House " $\mathrm{Z}$ " is the best option, because the price is lower for house " $\mathrm{Z}$ " than house " $\mathrm{M}$ " and the square footage is the same for both houses. But if one examined the details closer one would realize that house "Z" has an inferior feature. In fact, when one calculates taxes and Home Owners Association (HOA) fees, option "Z" is just as expensive as option "M." Moreover, house "Z" would cost more over the years because of the higher taxes and HOA fees. 
To reiterate, within the four purchasing scenarios given to each participant, two scenarios were house purchasing scenarios familiar to older adults and the other two were cell phone purchasing scenarios familiar to younger adults. With regard to the housing scenarios, each participant received one house purchasing scenario that required more complex inferencing and another house purchasing scenario that required little inferencing and had more explicitly stated information. Likewise, participants received two cell-phone scenarios. One of the two cell phone scenarios required inferencing and the other scenario had explicitly stated information. In each of the aforementioned scenarios, participants were instructed to select the better of the two options.

A latin square counterbalancing procedure was used to make sure that each option occurred in each ordinal position. That is, each scenario occurred equally in the first, second, third, and fourth position across participants. Also, the order in which the better option appeared (e.g., left or right side of the screen) within each scenario was counterbalanced across participants by reversing the order. Thus, the best option occurred equally on the left and right side of the screen.

In the second phase of the study, each individual option from the four purchasing scenarios was presented individually. A Likert evaluation scale was used for participants to rate the quality of the individual product options. The scale ranged from " 1 " indicating a low quality option to "7" indicating a high quality option. This phase of the study was included to check to see if participant choice selection in choosing between two scenarios was consistent with their ratings of each individual scenario. 
The experiment was administered on a windows based computer and took place in a quiet testing room. Older adults used a laptop as the experiment was carried out at various testing sites. The stimuli were presented via Superlab 4 Software Program.

Demographic questionnaire. A questionnaire was used to gather general information about the participants. This information was used to determine age, sex, health, and education. This Questionnaire is included as Appendix B.

$W A I S-R$. The vocabulary section of the WAIS-R test was used to test the verbal ability of each participant. The Wechsler Adult Intelligence Scale-Revised, a well established verbal ability test (Aronson \& Vroonland, 1993) was given to determine if the young and older adults have equal verbal intelligence. The internal consistency reliability of the WAIS-R has been shown to have an alpha ranging from $\alpha=.84$ to .90 . The Pearson test-retest reliability has also been shown to have a range of $r=.71$ to .86 (Hopp \& Dixon, 1997).

Trail Making Test. A Trail A and a Trail B working memory test was used to measure working memory. The test was given in two parts: part A and part B. Part A required the participant to draw a line connecting consecutive numbers from 1 to 25 . In part B participants were asked to perform the same action as part A but now had to connect alternating numbers and letters in sequence. Each trial was timed. Results were compared against average scores of 29 seconds for Trail A and 75 seconds for Trail B. Over 78 seconds was considered deficient in Trail A and 273 seconds was considered deficient in Trail B. (Arbuthnott \& Frank, 2000). The internal consistency reliability of the combined Trail Making Test has been shown to have a reliability rating of $r=.92$. The test-retest reliability has been shown to be $r=.84$. The composite Interrater reliability has also been shown to be very high $(r=.99)$ (Strauss, Sherman, Spreen, 2006). 
Mini-Mental State Examination. The Mini-Mental State Examination (MMSE) is a cognitive screening exam (Aronson \& Vroonland, 1993; Axelrod, Goldman and Henry, 1992; Folstein, Folstein and McHugh, 1975). This test was given to older adults and was used to screen out those individuals who might have dementia (those scoring less than twenty four on the MMSE). The results of these participants (i.e., those scoring below 24) were excluded to ensure that only healthy older adults were included in the study. The internal consistency reliability of the MMSE has been shown to have an alpha ranging from $\alpha=.90$ to .96 for older adults over 65 years of age while the Pearson test retest reliability has also been shown to have a range of $r=$ .89 to .99 (Hopp \& Dixon, 1997). The same Hopp \& Dixon study showed a construct validity for the MMSE in relation to the WAIS-R with a range of $r=.36$ to .39 .

Need for Cognition scale. The Need for Cognition scale (Cacioppo \& Petty, 1982), which measures the tendency of individuals to engage in and enjoy thinking, was also used. Participants were asked to complete the shorter 18 question version of this scale as opposed to the original 34 question version. This scale has been shown to have a reliability of $\alpha=.90$ (Cacioppo, Petty, \& Chuan Feng, 1984). This scale was included to determine if decision making ability (i.e., choosing a high quality option over a low quality option) is affected by need for cognition. That is, will individuals with high need for cognition be more likely to make decisions based on quality than people with a low need for cognition?

Multiple Resources Questionnaire. The Multiple Resources Questionnaire (MRQ) (Boles \& Adair, 2001) was used to assess participants' opinions of how much cognitive resources they used for specific processes in performing the decision making task. The MRQ has 17 processes on the questionnaire in which participants are asked to rate on a scale of $0-100$ how much of their cognitive resources they think they used in performing that process. The 
MRQ has been shown to moderate interrater reliability, ranging from $r=+.57$ to +.83 (Boles, Bursk, Phillips and Perdelwitz, 2007). Data from this questionnaire were collected for younger adults. In the above mentioned pilot study, I found that older adults had difficulty comprehending the questions, thus, I did not collect data on this measure from the older participants.

\section{Procedure}

There were two phases to the experiment. During phase 1, participants made purchasing decisions on the computer screen. During phase 2, participants rated each purchasing option individually. Both phases of the experimental procedure are described in more detail below.

Phase 1. Each participant was run independently. The experimenter remained in the room to answer any questions that came up and provided clarification when needed. Both younger and older participants were given a consent form to read. Older adults were required to sign the form. Once participants agreed to continue with the experiment, the experimenter explained the procedures and answered participants' questions. Participants were told that they would be required to read purchasing scenarios that would be presented on the computer screen. They were told that on each trial they would be presented with two options presented side by side. Their task would be to decide which of the two options in each purchasing scenario provided the best value for money. Once participants clearly understood the task, the experiment began.

When the participant pressed the space button, indicating that he or she was ready to begin the experiment, the instructions appeared on the screen. Participants were told to push the space bar when they were ready for the first trial. As indicated earlier, at this point, the purchasing scenarios appeared on the screen. Participants were given unlimited time to decide 
which of the two options was the best value for the money. After a selection was made, participants received a prompt thanking them for their selection before the next of the four purchasing scenarios was presented.

Phase 2. Following the choice selection of the four purchasing scenarios, the second phase of the experimental procedure began. At this point, participants were presented with the eight individual purchasing options one at a time in a new random order and asked to rate the quality of each option. An evaluation scale at the bottom of the screen below each scenario was presented to remind the participant of the rating scale. The participant typed in a number that indicated his or her rating of the product option. Each option remained on the computer screen until an evaluation had been entered for that option. Once the product had been evaluated, a thank you message was displayed and the next purchasing option appeared on the screen, remaining there until it had been evaluated. This procedure occurred until all eight products had been evaluated by the participant. Younger adults were then given the Multiple Resources Questionnaire to complete. All participants were then given the Need for Cognition Scale to complete. Following this, the Trail Making Test (Trail A and Trail B) to examine working memory was administered. The participants were then asked to complete a demographic questionnaire after which the Mini-Mental State Examination (MMSE) was administered to older adults. For the last task, participants received the WAIS-R vocabulary test.

After all aspects of the experiment were completed, the participants were debriefed on the experiment and told the purpose of the experiment. Any questions that participants had were answered. The participants were then given their compensation for participating in the study, either in the form of course credit for younger adults or monetary compensation for older adults. Older adults were asked to sign a receipt after receiving their $\$ 10$ compensation. Participants 
were then thanked for their participation and given information about who to contact if they had further questions or encountered any problems as a result of having participated in the study. 


\section{RESULTS}

\section{Demographics}

Of the 101 total participants, $75.2 \%$ were female and $24.8 \%$ were male. Results for means and standard deviations of age, vocabulary scores and the Trail Making Test scores are displayed in Table 3.

Table 3

Participant Mean and Standard deviation (SD) for age, vocabulary scores and Trail Making Test scores

\begin{tabular}{lccccc}
\hline & \multicolumn{2}{c}{ Younger adults $(\mathrm{n}=55)$} & & \multicolumn{2}{c}{ Older Adults(n=46) } \\
\cline { 2 - 3 } \cline { 5 - 6 } & Mean & SD & & & Mean \\
\hline Age & 18.71 & 1.45 & & & SD \\
Vocabulary & 26.05 & 10.86 & & 22.02 & 15.18 \\
Trail A & 22.14 & 4.88 & & 64.44 & 39.94 \\
Trail B & 48.92 & 22.96 & & 191.00 & 91.61 \\
\hline
\end{tabular}

\section{Psychometric Tests}

Verbal ability of participants was examined by administering the vocabulary section of the Wechsler Adult Intelligence Scale-Revised (Items 13-40). I compared the vocabulary scores of young and older adults by using an independent $t$ test. The difference in vocabulary scores between older adults $(\mathrm{M}=22.02, \mathrm{SD}=15.18)$ and younger adults $(\mathrm{M}=26.05, \mathrm{SD}=10.86)$, 
$t(99)=1.55, p=.12$ was not significant, which indicates that the two groups did not have markedly different levels of verbal intelligence.

Working memory was measured using results from the Trail A and the Trail B from the Trail Making Test. The maximum amount of time allotted for the combined Trail A and B is 5 minutes. It should be noted that three older adult participants were unable to complete Trail A and 16 older adults were unable to complete Trail B within the allotted time.

To determine if there were age differences in the Trail Making Test, independent t-tests were used to compare the performances of younger and older adults on both Trail A and Trail B. For Trail A, there was a significant difference between time taken for younger adults, $(\mathrm{M}=$ 22.14, $\mathrm{SD}=4.86)$ and older adults, $(\mathrm{M}=64.44, \mathrm{SD}=39.94), t(99)=-7.79, p<.01$ to complete the task. Errors in connecting the consecutive numbers or alphabet number combinations are not recorded but the time to correct errors is included in the total time to complete the task. For Trail $\mathrm{B}$, younger adults, $(\mathrm{M}=48.92, \mathrm{SD}=22.96)$ were significantly faster than older adults $(\mathrm{M}=$ 191.0, $\mathrm{SD}=91.61), t(99)=-11.10, p<.01$.

With regard to Trail B, there was one primary concern with regard to this task. The primary method of measuring performance is via reaction time and reaction time is supposed to be a measure of central or cognitive processing speed. However, this task requires some degree of motor dexterity in that participants have to draw lines between numbers and letters. I was concerned that older adults' slower speed was due in part to age-related changes in motor movement.

One way that I could address this issue is to determine the extent to which Trail B predicted performance in decision making after controlling for Trail A. Thus, I conducted a 
hierarchal regression of Trail A and Trail B scores entering Trail A scores first followed by Trail B scores to see how much of the variance Trail B accounted for in the regression model.

The results of the regression analysis show that Trail A scores were found to explain $1.9 \%$ of the variance in decision making performance. Trail B scores explained an additional 5.7\% of the variance in unfamiliar decision making scenarios after controlling for Trail A scores, R squared change $=.06, F$ change $(1,98)=6.07, p=.02$. The total variance explained by the model as a whole was $7.6 \%, F(2,98)=4.02, p=.02$.

\section{Results for Decision Making Task}

In this study, it should be noted that the products differed in quality but the prices of the products were the same. Thus, one product was always a better deal for the money than the alternative product. Thus, as mentioned earlier, a correct decision was one in which participants selected the higher quality option over the lower quality option.

It should also be noted that there were two types of decision making tasks in this study: implicit and explicit. As indicated earlier, my hypotheses were based solely on the implicit condition. The explicit condition was included in this study as a means of providing a manipulation check. Nevertheless, I analyzed the data in the explicit condition to determine if both groups of participants were taking the task seriously. I will first describe the analyses of the data in the implicit condition and then I will turn to analyses of the data in the explicit condition.

\section{Implicit Condition}

Table 4 displays decision making performance as a function of age and familiarity. As one can see, from Table 4, the majority of younger and older adults made correct decisions in the familiar condition-69\% and 78\% for younger and older adults respectively. However, in the 
unfamiliar condition, younger adults clearly outperformed older adults, with $76 \%$ of younger adults selecting the better option but only $50 \%$ of older adults selecting the better option. Table 4

Participant, Mean and Standard deviation (SD) for decision making quality in implicit familiar and unfamiliar scenarios

\begin{tabular}{lccccc}
\hline & \multicolumn{2}{c}{ Younger adults(n=55) } & & \multicolumn{2}{c}{ Older Adults(n=46) } \\
\cline { 2 - 3 } \cline { 5 - 5 } & Mean & SD & & Mean & SD \\
\hline Familiar & .69 & .47 & & .78 & .42 \\
Unfamiliar & .76 & .43 & .50 & .51 \\
\hline
\end{tabular}

To determine if age and familiarity affected decision-making quality, the data were subjected to a 2 Age (Young vs. Old) x 2 Familiarity (Familiar vs. Unfamiliar) ANOVA. As indicated earlier, only the implicit conditions were considered in the primary analysis. Alpha was set at .05. There were no significant main effects. However, there was a significant interaction between familiarity and age, $F(1,99)=8.46, M S E=1.58, p<.01, \eta_{p}^{2}=.08$.

To further examine this interaction, I conducted a follow up analysis in the form of paired $t$-tests comparing younger and older adults in the familiarity condition. Results indicate that there was no significant difference in younger adult performance for the familiarity condition, $t(54)=$ $-.89, p=.38$. There was a significant difference for older adults in the familiarity condition with older adults performing significantly better in the familiar condition $(\mathrm{M}=.78, \mathrm{SD}=.42)$ than the unfamiliar condition $(\mathrm{M}=.50, \mathrm{SD}=.51), t(45)=3.09, p<.01$. Therefore, the interaction indicates that younger adults' performance was unchanged regardless of domain while older adults performed significantly better in the familiar domain as supposed to the unfamiliar 
domain. That is, both groups of participants were able to make the "correct decision" a high proportion of the time in the familiar condition; in contrast, only the younger adults were able to make the "correct decision" a high proportion of the time in the unfamiliar condition.

\section{Explicit Condition}

The previous analyses indicated that there was a significant Age X Familiarity interaction in the implicit condition. I will next present the data from the explicit condition. As indicated earlier, in the explicit condition, it should have been very easy for participants to determine which of the two products provided the best deal for the money. In fact, the choice should have been so easy that I wasn't expecting any main effects or interactions. As stated earlier, the explicit condition was included as a manipulation check. Table 5 displays the means and standard deviations for the explicit condition as a function of Age and Familiarity. Table 5

Participant Mean and Standard deviation (SD) for decision making quality of familiar and unfamiliar purchasing scenarios in the explicit condition

\begin{tabular}{|c|c|c|c|c|}
\hline & \multicolumn{2}{|c|}{ Younger adults $(n=55)$} & \multicolumn{2}{|c|}{ Older Adults $(n=46)$} \\
\hline & Mean & SD & Mean & SD \\
\hline Familiar & .95 & .23 & .91 & .29 \\
\hline Unfamiliar & .96 & .19 & .87 & .34 \\
\hline
\end{tabular}

As one can see, performance was high across the board for both younger and older adults. This observation was supported by the results of a mixed factorial 2 Age (Young vs Older) X 2 Familiarity (Familiar vs Unfamiliar) ANOVA that was conducted on the data from the explicit condition. As expected there were no significant results. That is, there was no significant 
interaction between age groups and familiarity conditions, $F(1,99)=.73, M S E=.05, p=.40, \eta^{2}$ p $=.01$. There was also no significant main effect of familiarity, $F(1,99)=.12, M S E=.01, p=$ $.73, \eta_{\mathrm{p}}^{2}<.01$ or age, $F(1,99)=2.76, p=.10, M S E=.20, \eta_{\mathrm{p}}^{2}=.03$.

Recall that in addition to participants' making decision choices about which of two products was preferable in the first phase of the experiment, in the second phase of the experiment, participants rated the quality of each product as it was presented individually. To evaluate the participants' ratings of each individual product, a multivariate ANOVA was conducted on their responses. Age was used as the independent variable while participant ratings for the eight individual products were used as the dependent variable. I conducted this analysis because I wanted to examine the possibility that there were age differences in how people rated the individual products presented in the purchasing scenarios. Results show that the explicit cell phone purchasing scenario that was considered to be a poor purchasing choice showed a significant difference in participant responses between older adults $(\mathrm{M}=4.15, \mathrm{SD}=$ $1.94)$ and younger adults $(\mathrm{M}=2.98, \mathrm{SD}=1.43), F(1,99)=12.09, p<.01, M S E=34.31, \eta_{\mathrm{p}}^{2}=$ .11. Surprisingly, there was also a significant difference in ratings in the good quality implicit cell phone purchasing condition where older adults $(\mathrm{M}=5.85, \mathrm{SD}=1.28)$ were better able to rate the purchasing scenario as being of good quality than younger adults $(\mathrm{M}=5.33, \mathrm{SD}=1.16)$, $F(1,99)=4.60, p=.03, M S E=6.79, \eta_{\mathrm{p}}^{2}=.04$. No other ratings were found to be significantly different. The means are displayed in Figure 1.

\section{Working Memory, Aging, and Purchasing Decisions}

Thus far, my analyses have indicated that older adults are less likely than younger adults to make high quality purchasing decisions in situations in which the product is unfamiliar. In my hypotheses section, I predicted that age differences in purchasing decisions would be mediated 
via working memory. I examined this prediction via a series of hierarchical analyses. Because the unfamiliar implicit condition yielded the largest age differences in my earlier analyses, I chose this condition to represent my dependent variable in the regression analyses.

Figure 1

Younger and Older adult average scores for the individual scenario ratings

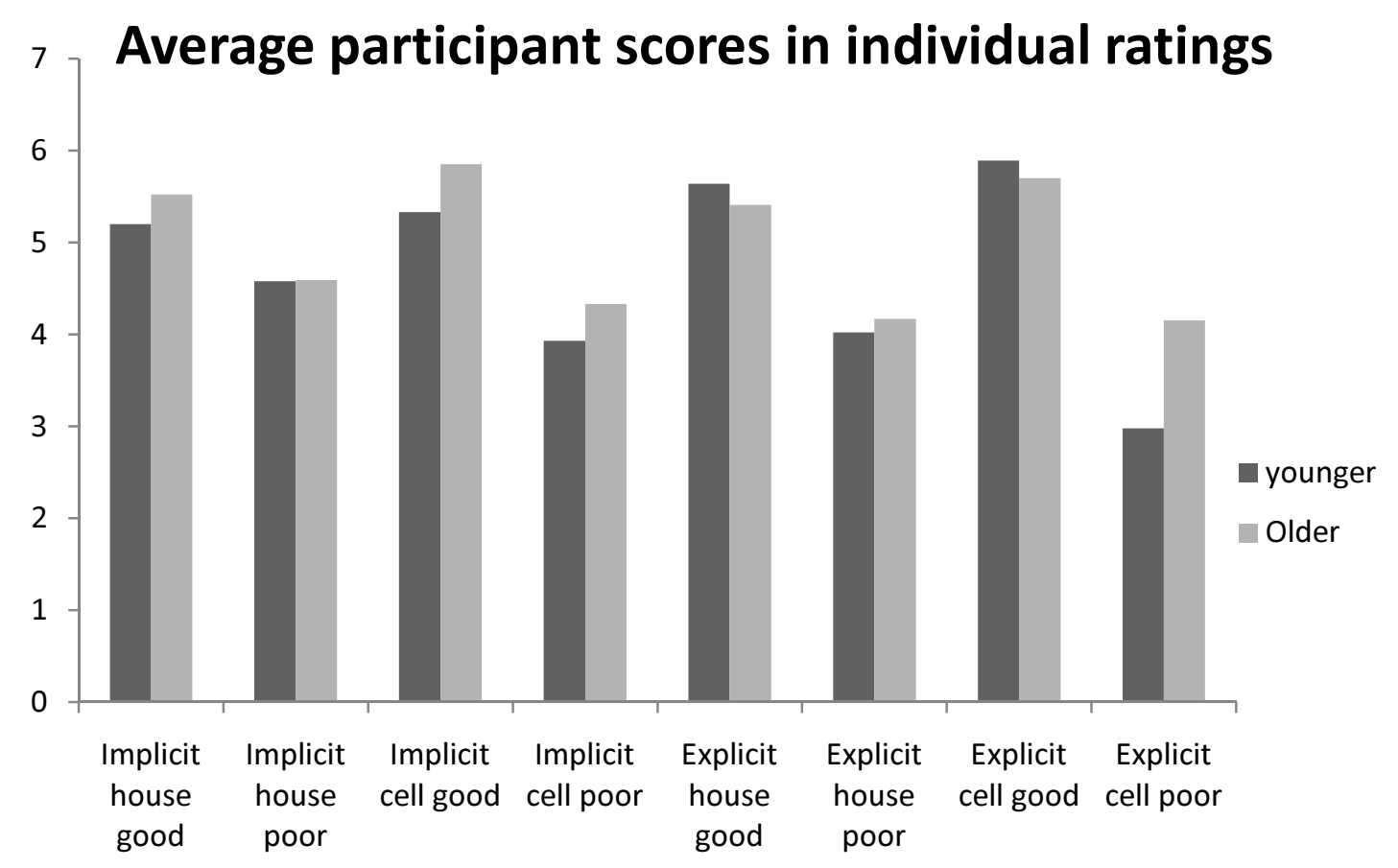

As indicated earlier, working memory was measured via Version A and B of the Trail Making Task. I chose the Trail B subtest of the Trail making test to represent working memory because prior research has shown that it is a good measure of executive processes. Based on prior research, I thought that executive processing would be particularly important for the decision making tasks examined in this study.

Before conducting my hierarchical analyses, I wanted to find out how much variance was accounted for by age in predicting purchasing decision performance when it was entered into the 
equation alone. Thus, I ran a simple regression on age with correct answers in the implicit unfamiliar condition being the dependent variable. Results showed that age accounted for $8.4 \%$ of the variance in the model, $F(1,99)=9.07, p<.01$. I then proceeded with additional regression analyses. The results of this analysis are displayed in Table 6 .

Table 6

Beta Coefficients, Beta significance, $R^{2}$ change, $F$ change and Significance $F$ change of percentage correct in unfamiliar decision making scenarios for age after controlling for working memory

\begin{tabular}{lccccc}
\hline \multicolumn{1}{c}{ Variable } & Beta Coefficient & Sig. coefficient & $R^{2}$ change & $F$ change & Sig. $F$ Change \\
\hline None & -.29 & $<.01$ & .08 & 9.07 & $<.01$ \\
Working memory & -.21 & .14 & .02 & 2.27 & .14 \\
\hline
\end{tabular}

In the first regression analysis, I wanted to examine the possibility that age would no longer be significant in predicting purchasing decision performance after controlling for working memory. At step 1of the first analysis, the scores from Trail B were entered and found to explain $6.8 \%$ of the variance in decision making performance. At step 2 of the first analysis, the age of participants was entered. Age explained an additional $2.1 \%$ of the variance in unfamiliar decision making scenarios after controlling for the working memory, R squared change $=.02, F$ change $(1,99)=2.27, p=.14$. The total variance explained by the model as a whole was $8.9 \%$, $F(2,98)=4.77, p=.01$. Interestingly, when working memory was not controlled, Age explained $8.4 \%$ of the variance, a significant portion of the variance associated with purchasing decisions. However, the amount of variance explained by Age was reduced by more than $50 \%$ when working memory was controlled resulting in age no longer making a significant contribution in 
explaining the variance associated with purchasing decisions. Thus, working memory at least partially mediated the relation between age and purchasing decision performance. The results of this analysis are displayed in Table 6.

To investigate the possibility that Need for Cognition moderated age effects within the unfamiliar condition, I created four groups of participants based on their scores on the Need for Cognition Measure. Based on a median split for each age group, I assigned half of the younger and older adults to a high need for cognition group and the other half were assigned to the low need for cognition group. The percent correct for the unfamiliar condition as a function of Age and Need for Cognition are displayed in Table 7. One can see that Need for Cognition improved performance overall, but this variable does not seem to moderate age effects. Upon reviewing Table 7, it appears that need for cognition improves performance equally for younger and older adults. These observations were supported by a 2 Need for Cognition (Hi. Vs. Low) x 2 Age (Young vs. Old) ANOVA. The ANOVA revealed no hint of an interaction between age and Need for Cognition $(\mathrm{F}<1)$.

Table 7

Participant Mean and Standard deviation (SD) for decision making quality in unfamiliar implicit scenarios with a median split of Need for Cognition scores

\begin{tabular}{lccccc}
\hline & \multicolumn{2}{c}{ Younger adults(n=55) } & & \multicolumn{2}{c}{ Older Adults(n=46) } \\
\cline { 2 - 3 } & Mean & SD & & Mean & SD \\
\hline Low Need For Cognition & .70 & .47 & & .43 & .51 \\
High Need for Cognition & .82 & .39 & & .57 & .51 \\
\hline
\end{tabular}


Finally, a hierarchical multiple regression was also conducted to examine the ability of age to predict participant scores in the unfamiliar implicit condition after controlling for scores from the Trail B test and controlling for scores from the Need for Cognition Measure. At step 1of this final analysis, the scores from the Trail B were entered and found to explain $6.8 \%$ of variance in unfamiliar decision making scenarios. At step 2 of this final analysis, combined Need for Cognition scores were entered and found to explain an additional $1.9 \%$ of variance in unfamiliar decision making scenarios. The age of participants was entered in step 3. Age explained an additional $2.1 \%$ of the variance in unfamiliar decision making scenarios after controlling for the scores from the Trail B and the total Need for Cognition scale totals, $\mathrm{R}$ squared change $=.02, F$ change $(1,97)=2.23, p=.14$. The total variance explained by the model as a whole was $10.7 \%, F(3,97)=3.88, p=.01$.

The results of the final regression analysis are displayed in Table 8. When compared to the results of the regression in which age is entered into the equation alone, one can see that age explains a significantly smaller proportion of the variance in predicting decision making performance when it is entered into the equation after working memory and need for cognition. In fact, age goes from explaining $8.4 \%$ of the variance associated with decision making performance when it is the only variable in the equation to $2.0 \%$ of the variance when it is entered after working memory and need for cognition. These results indicate that the relationship between age and purchasing decision making performance was mediated in large part by working memory. 
Table 8

Coefficients, significance, Increment in $R^{2}, F$ change and Significance of $F$ change for percentage correct in unfamiliar decision making scenarios affected by age after controlling for working memory and Need for cognition

\begin{tabular}{lccccc}
\hline \multicolumn{1}{c}{ Variable } & Coefficient & Sig. coefficient & $R^{2}$ change & $F$ change & Sig. F Change \\
\hline Working memory & -.26 & .01 & .07 & 7.19 & .01 \\
Need for Cognition & .14 & .16 & .02 & 2.05 & .16 \\
Age & -.21 & .14 & .02 & 2.23 & .14 \\
\hline
\end{tabular}

Table 9 presents a correlational matrix, displaying the extent to which variables such as Need for Cognition, Age, and Working Memory (as measured by Trail B) are correlated with each other. There are a few noteworthy points from Table 9. First, interestingly, Age does not correlate highly with Need for Cognition. Second, there is only a very modest correlation between Need for Cognition and Working Memory. Third, there is a strong correlation between Age and Trail B, .75.

Table 9

Intercorrelations Amongst Unfamiliar decision scores, Need for Cognition, Trail B and Age

\begin{tabular}{lcccc}
\hline \multicolumn{1}{c}{ Measure } & Unfamiliar Situations & Need for Cognition & Trail B & Age \\
\hline Unfamiliar Situations & - & .15 & $-.26^{* *}$ & $-.27^{* *}$ \\
Need for Cognition & - & -.04 & -.04 \\
Trail B & - & - & $.75^{* *}$ \\
Age (Young, Old) & & & - \\
\hline
\end{tabular}

**Correlation is significant at .01 level (2-tailed) 


\section{DISCUSSION}

The main purpose of this study was to examine age-related changes in decision-making ability across familiar and unfamiliar domains. I predicted that older adults would have the most difficulty making optimal decisions in conditions in which they had to evaluate unfamiliar products. I also predicted that age differences in performance in the unfamiliar condition would be at least partially mediated via working memory ability. In addition to predicting age differences in the unfamiliar condition, I predicted age constancy in the familiar condition. For the most part, the data were consistent with my predictions. I will now discuss each of these issues in more depth.

Age Effects in the Unfamiliar Decision-Making Condition

As indicated above, decision-making ability in this study appeared to be highly dependent upon working memory capacity. Working memory was measured in this study via the Trail-Making Task. In examining the results of the working memory performance of younger and older adults Trail A and Trail B scores, it is clear that working memory significantly decreases with age especially with regard to Trail B, a measure of executive processing (Libon et al., 1994). The fact that there were age differences in a measure of executive processing has important implications for this study because the current study is heavily dependent upon executive processing.

A component of executive processing is prioritizing and suppressing irrelevant information. In the current study, participants had to compare two products by reading over both purchasing scenarios describing the product. After reading the purchasing scenarios participants 
had to decide which details were important for assessing the quality of the product and which details should be ignored. In some instances, this comparison process required higher order reasoning skills and the ability to suppress information that was irrelevant. For example, in the cell phone condition (displayed in Appendix A), participants had to realize that the "minutes feature" was essentially the same for both purchases in that there was no difference between unlimited minutes and 4000 minutes. Thus, they should not pay attention to that feature. Instead, they should focus on other features that actually varied across products. It would take executive processes to "read between the lines" and to employ the appropriate strategy for evaluating the two products.

Along the same line, participants had to make inferences. That is, they had to realize that 4000 minutes actually means "unlimited minutes." Not surprisingly, older adults appeared to have more difficulty making appropriate inferences when evaluating unfamiliar products than younger adults. This is consistent with other research that shows that older adults have difficulty initiating spontaneous inferences (Hamm \& Hasher, 1992; Till and Walsh, 1980) due to decreased working memory capacity (Zacks \& Hasher, 1988) and that older adults have difficulty suppressing irrelevant information (Hamm \& Hasher, 1992; Lustig, Hasher and Tonev, 2001).

Evidence of the importance of the effect of working memory on decision making of older adults can be seen in the regression analyses examining the impact of age and working memory on decision-making capability. A series of hierarchical regression analyses were conducted to determine the extent to which age explained the variability in decision making ability after controlling for variables such as working memory. These analyses showed that age explained a significant amount of the variance when it was entered into the equation first. However, when 
working memory was entered first in the model and age was entered second, working memory accounted for greater variance in the model than age. This is clear evidence of how working memory is able to mediate the relationship between age and decision making.

In addition to working memory mediating the relation between age and decision making, I examined the extent to which Need for Cognition moderated age effects in the unfamiliar condition. One of the reasons that Need for Cognition might affect decision making ability is that older adults in general become cognitive misers as they age (Morris, Woo, and Singh, 2005). Thus, many older adults, especially those with low needs for cognition might not be motivated to deliberate when confronted with decision making in an unfamiliar situation. Given that these older adults do not enjoy deliberating anyway, they might be tempted to use a heuristic when confronted with an unfamiliar situation. On the other hand, older adult participants with high need for cognition might be motivated to deliberate when faced with an unfamiliar decision. This latter group of older adults enjoys analytical reasoning and thus this group might enjoy the challenge of making a decision about an unfamiliar phenomenon. Given the variability among older adults with regard to this variable, I was interested in exploring the possibility that older adults with a strong Need for Cognition might be more likely to use the elaborative route than older adults with a low Need for Cognition. I conducted an ANOVA for each age group after performing a median split based on Need for Cognition to separate participants into high and low Need for Cognition Groups. I found that individuals with a high Need for Cognition in each age group performed better than individuals with low need for cognition. However, I didn't find that Need for Cognition interacted with Age. To examine the combined effect of age, working memory and Need for Cognition I conducted multiple regressions with working memory, Need for Cognition and age in the same hierarchal model. When age was entered into the model after 
working memory and need for cognition, age no longer made a significant contribution in explaining the variance associated with decision performance. To summarize, I found that younger adults performed better than older adults in the unfamiliar domain and that the age difference was mediated by working memory capacity.

\section{Familiar Condition}

Thus far, I have focused on the condition in which younger adults outperformed older adults - the unfamiliar condition. However, when one examines the familiar condition, one can see that there are some positive findings from this line of research. This work shows that there were no age differences when the scenario focused on purchasing a house. This of course is due to the fact that the house purchasing scenario is familiar to older adults. Based on the pilot study, it is significantly more familiar to older adults than younger adults. Importantly, these results suggest that older adults could engage in elaborative processing and "read between the lines" when they had an established schema on which they could rely upon.

There is other published research showing that older adults can make decisions that are just as good as younger adults' under certain circumstances. For example, Leclerc and Hess (2007) have conducted research that indicates that older adults are at least as good as younger adults in making social inferences. They attribute older adults' ability to make superior social judgments, despite age differences in working memory, to older adults having "social expertise." That is, older adults' years of making social judgments allows them to evaluate people at a deeper level than younger adults and gives them an advantage when having to make social inferences. Kim \& Hasher (2005) also have found that older adults are able to make better decisions than younger adults when it is possible for older adults to follow simple rules in decision making. 
Thus, there is a growing body of literature indicating that age differences in decision making are attenuated in circumstances in which older adults have past experiences with the options being considered and/or when the decision-making task is not too complex. However, in unfamiliar domains, without the essential working memory resources, older adults are likely to engage in the peripheral route of persuasion in complex decision making situations.

Further research is required to determine what other elements associated with aging are having an effect on decision making ability of older adults. Hopefully, the additional research would allow investigators to develop adaptive strategies that will help older adults improve their decision making abilities in unfamiliar domains.

Finally, it is interesting to notice that when comparing results regarding purchasing scenarios types, both age groups made significantly better decisions in regard to the house purchasing scenario as opposed to the cell phone purchasing scenario. While this was expected for older adults because they were familiar with the house purchasing scenario, it was surprising for younger adults in that they were more familiar with cell phone purchasing scenarios.

It could be argued that the disparity in scores between cell phones and houses could be attributed to the fact that houses cost significantly more than cell phones and because of this, both younger and older adults have greater motivation to make the best purchasing decision for this costly product. They are therefore investing more cognitive resources hence the higher quality decision making rating for houses than cell phones.

This argument could be countered with the fact that although the house purchase is significantly more costly than the cell phone purchase, the cell phone was chosen to represent the familiar product of younger adults because for the average younger adult participant with an average age of 18.71 years, and limited financial resources, the cell phone purchase was 
considered to be a significant purchase within their financial realm. The cell phone would have a greater impact and importance on younger adult lives than a house purchase would have had. Therefore, while a house purchase may be financially more costly and seem to merit greater care, a cell phone purchase for a younger adult is more practically attainable, immediate and significantly relevant to their lives and should therefore merit greater care in the decision making process of younger adults.

One possibility is that the cell phone was just more difficult in that it contained a lure that was particularly difficult for younger and older adults to ignore, (i.e., the lure "unlimited minutes). Thus, the familiar condition for the younger adults was a bit more difficult than the familiar condition for the older adults. Thus, the performance in both groups might have been a bit higher in the conditions that included the cell phone scenario without such a salient lure in the poor quality cell phone scenario.

However, the important finding in this study is that younger adults performed above chance in a decision making task in an unfamiliar domain, in fact their performance was equal to that of older adults, despite their lack of experience. On the other hand, older adults' performance in an unfamiliar domain was at chance. With respect to the familiar domains, older adults performed at least as well as younger adults, despite the fact that older adults had less working memory resources than younger adults. 


\section{CONCLUSION}

To summarize, it is clear, as stated in previous research that there are age-related changes in working memory resources (Moye \& Marson, 2007). However, when older adults have experience within a domain, their experience allows them to make up for their cognitive deficits in decision making in comparison with younger adults. This is good news for older adults who have greater experiences in a wide variety of domains due to their life experiences.

Despite the fact that older adults are able to compensate for working memory deficits in familiar domains, older adults appear to lack the cognitive resources (Hess, Germain, Rosenber, Leclerc and Hodges, 2005) to compensate for age-related changes in cognitive resources such as working memory in unfamiliar domains. In the current study, this age-related decline in cognitive resources resulted in older adults' being unable to make optimal decisions in unfamiliar domains. 


\section{REFERENCES}

Axelrod, B., Goldman, R., Henry, R. (1992). Sensitivity of the mini-mental state examination to frontal lobe dysfunction in normal aging. Journal of Clinical Psychology, 48, 68-71.

Arbuthnott, K., \& Frank, J. (2000). Trail making test, part B as a measure of executive control: Validation using a set-switching paradigm. Journal of Clinical \& Experimental Neuropsychology, 518.

Aronson, H., \& Vroonland, J. (1993). The residual cognitive competence of elderly females. Journal of Clinical Psychology, 724-731.

Baddeley, A. D., \& Hitch, G. J. (1974). Working Memory. In G. Bower (Ed.), The psychology of learning and motivation, Vol. VIII, 47-90, New York: Academic Press.

Boles, D, B., \& Adair, L, P. (2001). The Multiple Resources Questionnaire (MRQ). In Proceedings of the Human Factors and Ergonomics Society $45^{\text {th }}$ Annual Meeting (pp.1790 - 1794). Santa Monica, CA : Human Factors and Ergonomics Society.

Boles, D. B., Bursk, J. H., Phillips, J. B., \& Perdelwitz, J. R. (2007). Predicting dual-task performance with the multiple resource questionnaire. Human Factors, 49, 32-45.

Brébion, G., Ehrlich, M. F., Tardieu, H. (1995). Working memory in older subjects: Dealing with ongoing and stored information in language comprehension. Psychological Research, 58, 225-232.

Cacioppo, J., \& Petty, R. (1982). The need for cognition. Journal of Personality and Social Psychology, 42, 116-131.

Cacioppo, J., \& Petty, R. (1984). The elaboration likelihood model of persuasion. Advances in Consumer Research, 11, 673-675.

Cacioppo, J., Petty, R., \& Chuan Feng, K. (1984). The Efficient Assessment of Need for Cognition. Journal of Personality Assessment, 48, 306.

Christensen, T., Ascione, F., Bagozzi, R. (1997). Understanding how elderly patients process drug information: A test of a theory of information processing. Pharmaceutical Research, $14,1589-1596$.

Cohen, G. (1981). Inferential reasoning in old age. Cognition, 9. 59-72. 
Connelly, S., Hasher, L., \& Zacks, R. (1991). Age and reading: The impact of distraction. Psychology and Aging, 6, 533-541.

Estevez, A., \& Calvo, M. (2000). Working Memory Capacity and Time Course of Predictive Inferences. Memory, 8, 51-61.

Finucane, M., Mertz, C., Slovic, P., \& Schmidt, E. (2005). Task Complexity and Older Adults' Decision-Making Competence. Psychology and Aging, 20, 71-84.

Finucane, M. L., Slovic, P., Hibbard, J., Peters, E., Mertz, C. K., \& MacGregor, D. G. (2002). Aging and decision-making competence: An analysis of comprehension and consistency skills in older versus younger adults considering health-plan options. Journal of Behavioral Decision Making, 15, 141-164.

Folstein, M.F., Folstein, S.E. \& McHugh P.R. (1975) Mini-Mental State: A practical method for grading the cognitive state of patients for the clinician. Journal of Psychiatric Research, 12, 189-98.

Hamm, V. P., \& Hasher, L. (1992). Age and the availability of inferences. Psychology and Aging, 7, 56-64.

Hartman, M., \& Hasher, L., (1991). Aging and Suppression: Memory for Previously Relevant Information. Psychology and Aging. 6, 587-594.

Hasher, L., \& Zacks, R. (1979). Automatic and effortful processes in memory. Journal of Experimental Psychology: General, 108, 356-388.

Hess, T., Germain, C., Rosenberg, D., Leclerc, C., \& Hodges, E. (2005). Aging-Related Selectivity and Susceptibility to Irrelevant Affective Information in the Construction of Attitudes. Aging, Neuropsychology, and Cognition, 12, 149-174.

Hopp, G., \& Dixon, R. (1997). Longitudinal and psychometric profiles of two cognitive status tests in very old adults. Journal of Clinical Psychology, 53, 673-686.

Johnson, M. (1990). Age Differences in Decision Making: A Process Methodology for Examining Strategic Information Processing. Journal of Gerontology: Psychologial Sciences. 45, 75-78.

Johnson, M. S., \& Drungle, S.C. (2000). Purchasing Over-The-Counter Medications: The Influence of Age and Familiarity. Experimental Aging Research, 26, 245-261.

Kim, S. \& Hasher, L. (2005). The attraction effect in decision making: Superior performance by older adults. Quarterly Journal of Experimental Psychology, 58A, 120-133.

Leclerc, C., \& Hess, T. (2007). Age differences in the bases for social judgments: tests of a social expertise perspective. Experimental Aging Research, 33, 95-120. 
Lemaire, P., Arnaud, L., \& Lecacheur, M. (2004). Adults' Age-Related Differences in Adaptivity of Strategy Choices: Evidence From Computational Estimation. Psychology and Aging, $19,467-481$.

Libon, D., Glosser, G., Malamut, B., Kaplan, E., Goldberg, E., Swenson, R., et al. (1994). Age, executive functions, and visuospatial functioning in healthy older adults. Neuropsyhology, 8(1), 38-43.

Lustig, C., Hasher, L., \& Tonev S.T. (2001). Inhibitory control over the present and past. European Journal of Cognitive Psychology, 13, 107-122.

Mata, R., Schooler, L., \& Rieskamp, J. (2007). The Aging Decision Maker: Cognitive Aging and the Adaptive Selection of Decision Strategies. Psychology \& Aging, 22, 796-810.

Meyer, B., Talbot, A., \& Ranalli, C. (2007). Why older adults make more immediate treatment decisions about cancer than younger adults. Psychology and Aging, 22, 505-524.

Morris, J., Woo, C., \& Singh, A. (2005). Elaboration likelihood model: A missing intrinsic emotional implication. Journal of Targeting, Measurement \& Analysis for Marketing, 14, 79-98.

Moye, J., \& Marson, D. (2007). Assessment of decision-making capacity in older adults: An emerging area of practice and research. Journals of Gerontology Series B: Psychological Sciences \& Social Sciences, 62B, 3-11.

Polyak, L. (2000). The Center of attention. American Demographics, 22, 30-33.

Petty, R. E., \& Cacioppo, J. T. (1984). Source factors and the elaboration likelihood model of Persuasion. Advances in Consumer Research, 11, 668-672.

Reed, A., Mikels, J., \& Simon, K. (2008). Older adults prefer less choice than young adults. Psychology and Aging, 23, 671-675.

Roberts, M., Welfare, H., Livermore, D., \& Theadom, A. (2000). Context, visual salience, and inductive reasoning. Thinking \& Reasoning, 6, 349-374.

Rowe, J. W., \& Kahn, R. L. (1998). Successful aging. New York: Pantheon Books.

Sorce, P., Loomis, L., \& Tyler, P. (1989). Intergenerational Influence on Consumer Decision Making. Advances in Consumer Research, 16, 271-275.

Staudinger, U., \& Baltes, P. (1996). Interactive Minds: A Facilitative Setting of Wisdom-Related Performance?. Journal of Personality \& Social Psychology, 71, 746-762.

Strauss, E., Sherman, E, M, S., \& Spreen, O. (2006). A Compendium of neuropsychological tests. New York: Oxford University Press. 
Te'Teni-Harari, T., Lampert, S., Lehman-Wilzig, S. (2007). Information processing of advertising among young people: The elaboration likelihood model as applied to youth. Journal of Advertising Research, 47, 326-340.

Till, P. E., \& Walsh, C. A. (1980). Encoding and retrieval factors in adult memory for implicational sentences. Journal of Verbal Learning and Verbal Behavior, 19, 1-16.

Yoon, C., Laurent, G., Fung, H., Gonzalez, R., Gutchess, A., Hedden, T., et al. (2005). Cognition, persuasion and decision making in older consumers. Marketing Letters, 16, 429-441.

Van der Linden, M., \& Bredart, S. (1994). Age-related differences in updating working memory. British Journal of Psychology, 85, 145.

Wright, H. H., \& Newhoff, M. (2002). Age-related differences in inference revision processing. Brain and Language, 80, 226-239.

Zacks, R., \& Hasher, L. (1988). Capacity theory and the processing of inferences. In L.L. Light and D.M. Burke (Eds.), Language, memory, and aging (pp. 155-169). New York: Cambridge University Press. 
APPENDIX A

\begin{tabular}{|l|l|}
\hline House $\mathbf{Z}$ & House $\mathbf{M}$ \\
\hline 1800sq feet & 1800sq feet \\
\hline Single level & Single level \\
\hline 3 bedrooms & 3 bedrooms \\
\hline 3 bathrooms & 3 bathrooms \\
\hline $\begin{array}{l}\text { \$450/month Home Owners } \\
\text { Association fees }\end{array}$ & $\begin{array}{l}\$ 200 / \text { month Home Owners } \\
\text { Association fees }\end{array}$ \\
\hline Central air conditioning & Central air conditioning \\
\hline High countertops & High countertops \\
\hline$\$ 5000$ real estate taxes & $\$ 3000$ real estate taxes \\
\hline Carport & 2 car garage \\
\hline Sale price $=\$ 198,000$ & Sale Price $=\$ 209,000$ \\
\hline
\end{tabular}




\begin{tabular}{|l|l|}
\hline Cell Phone Z & Cell Phone M \\
\hline 4000 minutes & Unlimited minutes \\
\hline 400 text messages included & 20cltext message \\
\hline Easy to use & Easy to use \\
\hline $\begin{array}{l}\text { 3 megapixel Camera } \\
\text { included in phone }\end{array}$ & $\begin{array}{l}\text { 3 megapixel Camera } \\
\text { included in phone }\end{array}$ \\
\hline Bluetooth enabled & Bluetooth enabled \\
\hline MP3 player included & MP3 player included \\
\hline Large display & Large display \\
\hline Video enabled & Video enabled \\
\hline Unlimited internet access & 5clkb internet access \\
\hline Price $=\$ 110.00$ & Price $=\$ 110.00$ \\
\hline
\end{tabular}




\begin{tabular}{|l|l|}
\hline Cell Phone Z & Cell Phone M \\
\hline Unlimited minutes & Unlimited minutes \\
\hline 400 text messages included & 100 text messages included \\
\hline Easy to use & Easy to use \\
\hline $\begin{array}{l}\text { 3 megapixel Camera } \\
\text { included in phone }\end{array}$ & $\begin{array}{l}1.5 \text { mega pixel camera } \\
\text { included in phone }\end{array}$ \\
\hline Bluetooth enabled & Bluetooth enabled \\
\hline MP3 player included & No MP3 player \\
\hline Large display & Large display \\
\hline Video enabled & No video capability \\
\hline Unlimited internet access & Unlimited internet access \\
\hline Price $=\$ 110.00$ & Price $=110.00$ \\
\hline
\end{tabular}




\section{APPENDIX B}

\section{QUESTIONNAIRE}

Subj\#

Product familiarity questionnaire:

1. Please enter your age.

2. Please select your gender.

M F

3. Please select your education level.

4. How many times have you bought a cell phone?

5. How familiar are you with purchasing a cell phone?

12

Not familiar

2

3 Somewhat familiar
6 5 Very familiar

6. What features do you consider to be important when purchasing a new cell phone?

7. How many times have you bought a house?

8. How familiar are you with purchasing a house?

$\begin{array}{cccccc}1 & 2 & 3 & 4 & 6 & 7 \\ \text { Not familiar } & & \text { Somewhat familiar } & & & \text { Very familiar }\end{array}$

9. What features do you consider to be important when purchasing a new house? (free entry) 